\title{
De Vancouver (1982) a Viena (1999): La Educación a Distancia Toma Nuevos Rumbos
}

\author{
(From Vancouver (1982) to Vienna (1999): Distance Education Takes a New Course)
}

\author{
Miguel Casas Armengol \\ Universidad Nacional Abierta \\ (Venezuela) \\ LiLy Stojanovic Pomerhanz \\ Universidad Central de Venezuela \\ (Venezuela)
}

\begin{abstract}
RESUMEN: El análisis y comparación entre los trabajos y tendencias presentadas en las Conferencias de ICDE, Vancouver (1982) y Viena (1999) permiten observar aquí el vigoroso desarrollo y cambios de la Educación a Distancia, durante los últimos diecisiete (17) años del final de este Siglo XX. Viena (1999) discutió los avances sobre temas fundamentales presentados en Vancouver (1982), pero también, nuevos Temas tales como: Globalización; Políticas para el Desarrollo; Aprendizaje Virtual y Universidades Virtuales; Entrenamiento para el Trabajo y la Producción; y, Mercados y Mercadeo. La enorme expansión Mundial de la Educación a Distancia y la gran diversidad de Nuevas Tecnologías, hacen ahora imperativo que las Universidades a Distancia existentes ó por crearse, adopten estructuras y enfoques muy creativos, que les permitan una gran flexibilidad y capacidad para responder rápidamente a nuevas demandas y al mejor uso de modernos instrumentos y técnicas.
\end{abstract}

\author{
Avances en Educación a Distancia - Impacto de Nuevas Tecnologías - Reestructuración de Universidades a Distancia
}

\begin{abstract}
The analysis and comparison between the papers and tendencies presented on the two ICDE Conferences of Vancouver (1982) and Vienna (1999), had made possible visualize the vigorous development and important changes of Distance Education during these seventeenth (17) years. Vienna (1999) debated the advances of some fundamental themes presented at Vancouver (1982), but in addition, also some new themes as: Globalization; Policies for Development; Virtual Learning and Virtual Universities, Developing the Company and Workforce Training of the Future, and Markets and marketing. The huge world expansion of Distance Education and the diversity of new technologies available for education, make now indispensable that existing Distance Universities or projected ones, adopt very creative structures and focus, that make possible a great flexibility and capacity to answer rapidly to this new demands and also, to make a better use of modern instruments and technologies.
\end{abstract}

Progress in Distance Education - Impact of New Technologies - Restructuration of Distance Universities 


\section{INTRODUCCIÓN}

Una forma significativa para apreciar la evolución y progresos de muchas disciplinas científicas y profesionales es observar y comparar los aportes sucesivos que se presentan en los eventos mundiales de cada especialidad. Allí, mediante una observación longitudinal en tiempos suficientemente largos, se pueden apreciar con más claridad, los cambios de rumbo e innovaciones que consolidan determinadas tendencias. Es por ello, que en el caso de la Educación a Distancia, hemos considerado útil analizar la reciente Conferencia Mundial del International Council on Distance Education (ICDE-Viena 99), pero estableciendo previamente algunas comparaciones con la Conferencia del mismo ICDE, celebrada hace diecisiete años en la ciudad de Vancouver, Canadá.

\section{ICDE / VANCOUVER 1982. HITO SIGNIFICATIVO EN LA EVOLUCIÓN DE LA MODALIDAD A DISTANCIA}

La Conferencia de Vancouver 1982, al igual que otras anteriores, fue convocada inicialmente por el "International Council for Correspondence Education" (ICCE) y estuvo precedida por la reunión de New Delhi (India) en 1978, y un año más tarde, por la de la Open University de la Gran Bretaña, que celebraba diez años de existencia, mediante una Conferencia sobre Educación de Adultos a Distancia (CEAD).

La reunión de Vancouver representó un hito muy importante en la larga trayectoria del ICCE, que durante casi cuarenta años estuvo conformado principalmente por acreditadas Escuelas mundiales por Correspondencia. Sin embargo, en los últimos años anteriores a 1982, habían aumentado significativamente en el ICCE los miembros e instituciones provenientes de universidades y esto empezó a configurar distinciones cada vez mayores. entre los clásicos "estudios por correspondencia" y la recién llegada "educación a distancia", Asimismo, como consecuencia natural de la nueva influencia universitaria, empezó a abrirse un campo de investigaciones apropiadas para la nueva modalidad a distancia, que hasta ese entonces se había basado principalmente en una extensa práctica, pero sin mayor apoyo en principios científicos, teorías e investigaciones. Una consecuencia de la presión de estas nuevas fuerzas, fue que en la reunión de Vancouver 82, se aprobó que de allí en adelante el ICCE se transformaría en el "International Council on Distance Education", (ICDE), que sigue siendo hasta hoy día su denominación oficial.

En Vancouver 82 la concepción principal sobre Educación a Distancia giró alrededor del modelo desarrollado con gran éxito por la Open University, (OU) de la Gran Bretaña. En esa universidad los diseñadores y directivos de ella comprendieron oportunamente, que pese a la romántica idea inicial de crear una "Universidad del Aire", la tecnología disponible para aquel momento, limitaba las posibilidades operativas para llevar a cabo tal idea. Por consiguiente, el modelo de la OU se estructuró alrededor de un texto impreso de gran calidad en su contenido y con una diagramación sumamente creativa, tanto desde el punto de vista pedagógico como estético. Este elemento, se complementó con algunos recursos audiovisuales, principalmente a través de Radio y Televisión, aprovechando las importantes instalaciones y experiencias de la BBC Británica. La entrega oportuna de materiales, e informaciones, se facilitaron a través de un Correo Nacional de gran eficiencia. Finalmente, una red de Centros Regionales, coordinaba la compleja operación de numerosos orientadores y tutores, provenientes de una gran diversidad de instituciones que apoyaron a la Open University.

Dentro del modelo aquí descrito, conviene observar que la OU, para lograr aceptación en el exigente sector universitario británico, puso un notable empeño en que sus materiales fueran de una altísima calidad científica, y ello pese a su elevado costo de producción y al largo tiempo necesario para su diseño y producción. Tomado en cuenta este aspecto, durante las primeras etapas de esta universidad, ella no ofrecía carreras profesionales estructuradas y los alumnos disponían de una enorme flexibilidad para seleccionar los cursos deseados.

Un examen de los aportes presentados en Vancouver 82 refleja claramente el "estado del arte" mundial, de la Educación a Distancia para esa fecha. Como señalamos anteriormente, el modelo de la OU consciente o inconscientemente, era el dominante en la mayoría de las discusiones de la Conferencia y de lo que se trataba era de perfeccionarlo, adaptarlo, enriquecerlo y sobre todo, de proporcionarle un mejor soporte a través de la investigación y de la definición de sus bases teóricas. Sin embargo, todavía aparecieron allí algunos trabajos que expresaban una cierta añoranza por la creciente disminución de la importancia de la educación por correspondencia. No obstante, se perfilaron marcadamente en la Conferencia, dos posiciones contradictorias que se referían, la primera, a la supuesta "fragilidad" que muchas universidades tradicionales existentes le atribuían a este nuevo tipo de educación no-formal, que consideraban despectivamente como de "segunda clase" ("Learning at the Back Door", según la expresión irónica de Wedemeyer, 1981), y la segunda posición, a una visión prospectiva y optimista de la educación a distancia, como una futura gran fuerza moldeadora de sociedades en todo el mundo. Esta última posición se destacó especialmente, en relación con países de escaso desarrollo, para los cuales, 
la educación a distancia, se visualizaba que podría representar quizás, una de las pocas nuevas alternativas de gran impacto y viabilidad.

Sólo como una pequeña muestra de las principales orientaciones de la Conferencia de Vancouver (1982) mencionaremos para los Capítulos sobre Tendencias Internacionales, y Desarrollo Nacional: los trabajos de: Perraton (1982),"Distance Teaching North and South"; Baăth (1982), "From New Delhi to Vancouver"; Dat (1982) "Problems of Distance Education in Developing Countries"; y Peters (1982), "Continuing Education by Distance Study”. En el Capítulo sobre Aprendizaje a Distancia pueden señalarse: Coldeway (1982), "What Does Educational Psychology Tell Us About the Adult Learner at a Distance"; Penney (1982),"Content Analysis for Courses Design"; y Jevons (1982) "How Different is the Distance Student”. En el Capítulo sobre Soporte al Estudiante y Servicios Regionales, se destacan especialmente los trabajos de: Lewis (1982), "The Role of the Correspondence Tutor": y el de Mills \& Tate (1982) "Student Support Services in Continuing Education". Otros trabajos en diversos Capítulos se referían a temas varios tales como: Rumble (1982) "Responding to Economic Austerity: Can Economic Models of Distance Teaching Help Us? Howell (1982) "Read and Writte Better”; y Jenkins (1982) “Tell Me How to Writte”.

Mención aparte merece la atención prestada a los temas de Investigación y a las Contribuciones de los Medios y de la Tecnología. En relación con la Investigación se notó un creciente interés en ella, pero con muy pocos aportes que pudieran considerarse como verdaderas investigaciones. El trabajo teórico más importante fue el de Coldeway (1982), "Recent Research in Distance Learning”. Por otra parte, en lo concerniente a los Medios y la Tecnología, la mayoría de las escasas contribuciones se refirieron a los clásicos apoyos Audiovisuales para los Textos Escritos, especialmente mediante Radio, Televisión, Vídeo, Videodiscos y Videotexto. La Computación apareció tímidamente en lo relativo a Procesamiento de Datos, Procesadores de Palabras, y algunos experimentos iniciales sobre enseñanza asistida por computadores; en este sentido, los trabajos más representativos fueron: el de Baåth (1982), "Experimental Research on Computer-Assisted Distance Education"; y el de Bates (1982) "Trends in the use of Audio-Visual Media in Distance Education Systems".

En síntesis, podría concluirse que la Conferencia Vancouver (1982) constituyó una encrucijada muy decisiva para avanzar en la conceptualización de la nueva "Educación a Distancia", vista entonces como una Modalidad cada vez más distinta a la de la "Educación por Correspondencia". Así mismo, sirvió para comprender la necesidad de investigar y producir continuamente un nuevo cuerpo de conocimientos, teorías y principios, que proporcionaran un perfil más definido y sólido para esta Modalidad a Distancia. La Conferencia también resultó útil para empezar a percibir que la Educación a Distancia estaba llamada a jugar un papel futuro trascendental y creciente en el escenario mundial, porque su capacidad de resolver problemas de tiempo y espacio, la convertían en un instrumento sumamente apropiado y estratégico para sociedades desarrolladas, pero más aún, para aquellas de escaso desarrollo. Para esa fecha de la Conferencia las ideas giraron en gran parte, como ya se ha señalado, alrededor del "modelo bandera inicial "de la OU, y de las tecnologías entonces disponibles, que eran relativamente limitadas. Este modelo británico citado, sirvió de ejemplo y estímulo para crear numerosas universidades y programas a distancia en muchos países, con resultados no siempre felices, porque se ignoraron en muchos casos que las condiciones, objetivos y contexto socio-cultural de la OU no eran los mismos de otros países y situaciones. Por otra parte, también resultó difícil en la Conferencia, predecir con cierta precisión, el vertiginoso crecimiento futuro de las Tecnologías Informáticas-Telemáticas, que en menos de las dos décadas siguientes, hicieron posible transformar radicalmente muchos procesos de instrucción, pero especialmente, los de la Educación a Distancia.

\section{ICDE / VIENA 99. LA NUEVA FRONTERA EDUCATIVA: ENSEÑANDO Y APRENDIENDO EN UN MUNDO EN RED}

Después de diecisiete años y de siete sucesivas Conferencias Internacionales del ICDE, encontramos ahora en la XIX Conferencia de Viena (1999), que la Educación a Distancia que delineó la Conferencia de Vancouver (1982), ha experimentado desde entonces, cambios continuos sumamente importantes, que seguramente continuarán aún más, en el futuro que se iniciará desde el año 2000. El énfasis que en Vancouver puso la atención casi exclusivamente en las Universidades a Distancia, se amplía ahora hacia otros nuevos campos tales como: "Escuelas de varios niveles educativos", y la "Educación y Entrenamiento para el Trabajo y la Producción". Así mismo, surgen cada vez mayores dudas sobre la futura exclusividad de esta modalidad sólo para las Universidades especializadas a Distancia. En efecto, el movimiento mundial denominado "Convergencia", apunta a que en muy pocos años, muchas universidades convencionales, utilizarán cada vez más, la Enseñanza a Distancia, conjugándola funcionalmente con la Enseñanza Presencial. Los enemigos principales para el robustecimiento y progreso de las instituciones y programas a distancia, han sido y siguen siendo, la falta de: Definición, Calidad, Innovación y Pertinencia, en diversos casos. Por otra parte, se utiliza con demasiada frecuencia la etiqueta aparentemente novedosa, de "Educación a Distancia", para designar instituciones, cursos y prácticas de dudosa calidad educativa y carentes de los principios teóricos y elementos fundamentales de una verdadera educación a distancia. 
Tales programas, especialmente en las Sociedades de escaso desarrollo, no producen generalmente ni conocimientos, ni formación, y se orientan exageradamente, hacia la fácil concesión masiva de Títulos y Diplomas, que sólo ahogan el progreso social y científico.

Sin duda, el tema más destacado de la Conferencia Viena (1999), fue el de las "Nuevas Tecnologías". Esto ya venia ocurriendo progresivamente en las Conferencias anteriores, pero en Viena, este tema presentó un protagonismo impactante. Sin embargo, conviene aclarar que la importancia de los extraordinarios avances tecnológicos actuales, no reside tanto en la novedad, cantidad, diversidad y cambios de estos modernos equipos, sino en el hecho de que ellos ofrecen ahora la oportunidad de transformar profundamente los procesos de aprendizaje, creándose así un nuevo "Paradigma",que sustituya con ventajas, al presencial que se ha venido utilizando desde hace más de doscientos años. Sin embargo, debe apuntarse, que la sola aplicación mecánica de las más avanzadas tecnologías, sin el indispensable aporte de apropiados diseños y buenas estrategias instruccionales, no será capaz de producir los mejores resultados educativos.

Los temas dedicados exclusivamente a la Tecnología o a otros temas sustantivos, también apoyados claramente en elementos tecnológicos, conformaron una proporción abrumadora en todas las Sesiones Plenarias, Simposios, Sesiones Paralelas, Presentación de Pósteres, Sesiones de Demostración, y Actividades de Extensión, de la Conferencia. De ello puede deducirse que hoy día, ningún Programa o Universidad a Distancia pueden pretender ignorar, la necesidad de incorporar amplia y funcionalmente, un conjunto de avanzadas tecnologías para su organización y operación, pero tal imperativo no podrá resolverse sólo con la simple adquisición de numerosos equipos modernos, porque será indispensable considerar también la necesaria e importante distinción, que debe existir entre equipos y tecnologías, tal como lo aclara la definición de Naughton (1994) sobre Tecnología:

"Aplicación del conocimiento científico, y de otros conocimientos, a problemas concretos, mediante un conjunto integrado de estrategias, procesos y tareas prácticas, llevadas a cabo por organizaciones, que incluyen personas y equipos (tanto tradicionales como modernos)" (p.12).

\subsection{ESTRUCTURACIÓN DE LA CONFERENCIA}

La Conferencia se organizó alrededor de tres grandes Ejes:

U Eje U: CONSTRUYENDO LA UNIVERSIDAD DEL FUTURO

U Eje S: CONSTRUYENDO LA ESCUELA DEL FUTURO

U Eje T: DESARROLLANDO LAS COMPAÑIAS Y ENTRENANDO SU FUERZA DE TRABAJO DEL FUTURO.

En cada uno de estos Ejes se fijaron seis (6) Temas Principales, con igual denominación para cada uno los tres Ejes:

o Tema (a): Aprendizaje Abierto y Educación a Distancia como herramientas estratégicas para el Desarrollo.

o Tema (b): Los nuevos ambientes del Aprendizaje.

o Tema (c): Globalización de la Educación: Beneficios y Limitaciones.

o Tema (d): Políticas y Estrategias para el Desarrollo.

o Tema (e): Demoliendo las barreras mediante Educación y Entrenamiento.

o Tema (f): Mercados y Mercadeo.

Esta organización aquí esquematizada, hizo posible que dada la gran cantidad de documentos discutidos en la Conferencia y la simultaneidad de muchas presentaciones, cada participante pudiera concentrarse en el Eje y Temas que le resultaran de mayor interés. En lo relativo a este Informe, se analizarán especialmente los Temas y Actividades relativas al Eje (U) "CONSTRUYENDO LA UNIVERSIDAD DEL FUTURO". Para este propósito y dada la gran cantidad de contribuciones, destacaremos solamente aquellas que por su carácter indicativo y su contraste con las de Vancouver (1982), permitan formular posteriormente, observaciones útiles, para apreciar la evolución mundial de la Educación a Distancia. 
Aunque la discusión de los Temas específicos fijados para la Conferencia se desarrolló principalmente en las "Sesiones Paralelas" y en las "Sesiones de Pósteres", debe señalarse que también se obtuvieron aportes muy valiosos en las Conferencias Plenarias, Conferencias Especiales, Paneles y Demostraciones. Destacada mención merecen en este sentido:

Lu presentación de Otto Peters de la FernUniversität de Hagen (Alemania), titulada La Universidad del Futuro. Perspectivas Pedagógicas. En nuestra opinión, fue la contribución más importante para el Eje U "Construyendo la Universidad del Futuro" de esta Conferencia. Peters, ubica aquí el importante rol de las nuevas tecnologías, para la profunda transformación de la universidad tradicional actual, pero partiendo principalmente de los cambios pedagógicos concretos que requieren los nuevos procesos de aprendizaje y enseñanza, en las sociedades contemporáneas. Este es un enfoque mucho más preciso y profundo que las usuales digresiones superficiales sobre el potencial de las Nuevas Tecnologías y las necesidades de cambios en las universidades existentes (presenciales y a distancia).

4 La exposición de Marku Markkula, Secretario de la "Asociación Internacional para la Educación Continua en Ingeniería" (Finlandia), quien presentó: "Política Nacional de Aprendizaje por vida. Cómo implementar las estrategias de cambio". Esta singular presentación, intentó mostrar como orientar y definir un modelo con las estrategias y acciones requeridas para el cambio global de todo un sistema educativo nacional. Tomando como ejemplo su propio país, definió las guías y acciones allí utilizadas, para lograr convertirlo en muy pocos años, en una sociedad totalmente informatizada. Conviene agregar que el Dr. Markkula es también miembro del Parlamento Finlandés.

uv Juan Carlos Tedesco, Director del Instituto Internacional de Planeamiento Educativo de UNESCO en Buenos Aires, plantea en su trabajo "Educación y Tecnología de Información”, la polémica hipótesis siguiente: aunque reconoce que las nuevas tecnologías de la información están asociadas a cambios sociales, culturales y políticos de significativa profundidad, él sostiene "que son los actores sociales y sus orientaciones las que explican el surgimiento y desarrollo de las nuevas tecnologías" En este contexto insiste en que resulta importante analizar la relación entre educación y nuevas tecnologías, desde el punto de vista de su impacto en el proceso de socialización, y también el de su impacto en el proceso de aprendizaje de contenidos curriculares escolares.

14 La presentación altamente especializada de José L. Encarnaçao de la Universidad Técnica de Darmstadt (Alemania), se denominó: "iRedes del Conocimiento: Calificaciones Individuales en Organizaciones de Aprendizaje". El creciente uso mundial de tecnologías de Internet cambiará dramáticamente el uso del conocimiento: ese conocimiento será distribuido a muchas partes del mundo, accesible en forma omnipresente, y usado colaborativamente por equipos virtuales. Un nuevo Paradigma motorizado por las nuevas tecnologías, nos llevará a cambios constantes de los productos del conocimiento y abrirá grandes posibilidades para los escenarios innovadores donde se hará uso de tales conocimientos. Este nuevo término "iRedes del Conocimiento", refleja por consiguiente, lo relativo a los dominios del conocimiento distribuido, basado en Internet, las redes humanas de equipos virtuales, y un conjunto interconectado de elementos inteligentes.

Entre los diversos Paneles realizados durante la Conferencia, se destacó especialmente uno que planteó tres importantes preguntas, en lo relativo a Universidades y Programas a Distancia fundados hace tiempo, en varias partes del mundo: ¿Qué esperábamos? ¿Qué pasó? ¿Qué expectativas para el futuro? ("Expect-Happen-Hope"). Participaron cuatro especialistas mundiales de dilatada experiencia: Borje Holmberg, Suecia; Lord Perry of Walton, Gran Bretaña; Erling Ljosa, Noruega; y Miguel Casas Armengol, Venezuela. Resultó muy ilustrativo constatar las diferencias de enfoque y solución de las varias instituciones y países, frente a imprevistas y nuevas variables, surgidas en su proceso de evolución institucional.

En lo relativo al Tema (a) “Aprendizaje Abierto y a Distancia, como herramienta estratégica para el Desarrollo”, nos limitaremos a citar sólo algunos trabajos, para mostrar la orientación temática del conjunto de tales aportes: Evaluation of open university systems: a study of Indira Ghandhi National Open University (Satyanarayana Polu, India). Policy, practice and paradigm: how to develop a virtual university without loosing your shirt (Stephen Brown y otros, De Montfort University, Reino Unido). Latin American distance education universities: research for the future (Ramona Akl-Bittar, Universidad Nacional Abierta, Venezuela). The role of tutor development in a flexible learning system (Jean G. J. Grundling, Technikon SA, Sudáfrica). Technological training: from distance education to telematics (José Ramón Ortiz, Universidad Nacional Abierta, Venezuela). Distance education for corporate engineers (Yoshi Y., Japón). Implication of the use of cognitive strategies in instructional design for distance education in the Latin American context (Lily Stojanovic, Universidad Central de Venezuela, Venezuela). Critical issues to consider before committing your organization to developing on-line instruction (Victor H. Aulestia, University of Maryland, Estados Unidos).

El conjunto de trabajos presentados para este Tema (a), que incluyen también los reseñados en el párrafo anterior, muestran el vigor y consolidación de esta nueva modalidad educativa en las dos últimas décadas, y como su aplicación se extiende 
cada vez más a distintos sectores de la sociedad. Esto ha sido posible, por una parte, gracias a las numerosas investigaciones y estudios que le han dado a esta modalidad, un perfil más sólido y confiable y con mayores posibilidades estratégicas de expansión. Por otra parte, las nuevas herramientas tecnológicas han encontrado en la educación a distancia un campo especialmente apropiado para apoyarlo y darle una fuerza de penetración, que no tuvo en sus orígenes. Casos concretos de estas nuevas posibilidades, se encuentran en las tendencias hacia la virtualización de las universidades, el uso de la instrucción en línea, y de mejores apoyos tutoriales a través de la Telemática. Al mismo tiempo, por sus características de poder dar mejores respuestas a los problemas de espacio y tiempo, con costos generalmente más bajos, la educación a distancia puede irrumpir ahora con éxito en áreas masivas como la relativa a la culturalización de la ciudadanía, la extensión hacia niveles educativos distintos al universitario, y también en la formación, entrenamiento y actualización continuas, de Recursos Humanos para la producción

Con similar objetivo que en el párrafo anterior pero en lo concerniente al Tema (b) "Los nuevos ambientes del Aprendizaje", citaremos del amplísimo repertorio presentado, solamente los trabajos siguientes: Marrying on-campus teaching to distance teaching (Claus Unger y otros, FernUniversität, Alemania). Instructional techniques in the on-line classroom (University of Alberta, Canadá). Motivational experience for research (Miryam Flores, Universidad Nacional Abierta, Venezuela). The interactive paperless virtual class (Dam Lim, University of Minnesota, Estados Unidos). Advice and choice for students on-line: an open university perspective (David Sewart, Open University, Reino Unido). Student skills in a virtual university (Elena Barbera, Universitat Oberta de Catalunya, España). Toward a model that supports cooperative work in a virtual learning environment (Montse Guitert y otros, Universitat Oberta de Catalunya, España). Improvement of the production of research in distance graduate students: a case study (Ramón Escontrela Mao, Universidad Central de Venezuela, Venezuela). Teaching control theory by multimedia (Wolfram Laaser, FernUniversität, Alemania). Vocational education within the virtual campus (Ana Pages Santacana, Universitat Oberta de Catalunya, España). The virtual paradigm in higher education. Implications for quality, equity and relevance (José Silvio, Cresalc/Unesco, Venezuela). Staffing the virtual university: a training plan for developing faculty expertise for web-based courses (J.S. Boyce, University of Maryland, Estados Unidos). Assignments in a virtual university: The web-assign system (Hans-Werner Six y otros, FernUniversität Hagen, Alemania). Distance education: the fifth generation (James C. Taylor, University of Southern Queensland, Australia). From face-to-face to virtual tutoring: a way to improve tutorial support for the students (Maria J. Bermúdez, Universidad Nacional Abierta, Venezuela). Alternative quality criterion to evaluation in higher distance education (Beatriz Tancredi, Universidad Nacional Abierta, Venezuela).

Este Tema (b) "Los nuevos ambientes del aprendizaje", fue el que despertó el máximo interés de los participantes de la Conferencia. En efecto, el número de trabajos aceptados y discutidos para este tema (en sesiones paralelas y de pósteres) superó en mucho a cualquiera de los otros temas del evento. Pero esta característica no resulta sorprendente, porque existe en el mundo actual, dedicado a esta modalidad, el cada vez mayor convencimiento de que las condiciones cuasi-artesanales que presentaba la Educación a Distancia en sus inicios, deben experimentar ahora una radical transformación para poder acoger los nuevos Paradigmas tecnológicos de esta modalidad. Los numerosos trabajos presentados, giran principalmente alrededor de los conceptos de "aprendizajes virtuales y universidades virtuales". Ello incluye: consejos, destrezas y asignaciones para docentes y estudiantes en universidades de este nuevo tipo; trabajos cooperativos y educación vocacional en ambientes virtuales; experticia para Cursos en la Web; implicaciones del Paradigma virtual en la Educación Superior; criterios alternativos de calidad; motivaciones para la investigación y mejoramiento de la producción investigativa. Así mismo y dada la creciente importancia del movimiento mundial de "convergencia", se presentan también algunas propuestas sobre la inserción o "matrimonio" de la Educación Presencial con la Educación a Distancia.

En lo relativo al Tema (c) “Globalización de la Educación: Beneficios y Limitaciones”, algunas de las principales contribuciones fueron las siguientes: The global virtual university (John Tiffin y otros, The Global Virtual University, Nueva Zelanda). Assessing quality in the design of education of international virtual graduate programs. A new model of evaluation (Yolanda Gayol, The Pennsylvania State University, Estados Unidos). CAERENAD. An international networkpartnership in distance education (André-Jacques Deschenes, Télé-université, Canadá). An innovative response in Spain to formal and non-formal university education needs. Toward the third decade of open university life (Lorenzo García Aretio, UNED, España). International learning networks (Alejandro Ibarra, Nova Southeastern University, Estados Unidos). The practice of mediated and global communication and study-timing theory (Emilio Nogales, UNED, España). Open university students in continental Western Europe: a description of an ODL community organised across international boundaries (Peter Regan y otros, Open University, Reino Unido).

Aunque la Globalización es un fenómeno mundial relativamente reciente y referido a aspectos económicos, políticos y culturales macros, su importancia en aumento ya no puede ser ignorada por la Educación a Distancia, que por sus características, resulta especialmente adecuada para orientar esta visión de creciente interacción internacional. La mayoría de los trabajos presentados enfatizan las indudables ventajas del uso de la modalidad a distancia en la Globalización, pero hay muy pocos análisis sobre sus limitaciones y peligros, especialmente para sociedades de escaso desarrollo. 
El Tema (d) "Políticas y Estrategias para el Desarrollo" es analizado en trabajos como los siguientes: Distance education via electronic networks and the development of minority francophonie communities in Canada (Denis Carrier, University of Ottawa, Canadá). Distance education: a decisive force for the restructuration of Latin American university (Miguel Casas Armengol, Universidad Nacional Abierta, Venezuela). Networking for Europe? The role of open distance learning networks in promoting European competitiveness and cohesion (Kay Mac Keogh, Dublin City University, Irlanda). Partnership for academic programs in distance and open learning (Ashish Aganwal, Indira Gandhi National Open University, India). Bridges to global learning: a virtual academic library environment via the Internet (John D. Gaboury, William Paterson University, Estados Unidos). Cooperation in the diversity (Manuel Moreno Castañeda, Universidad de Guadalajara, México). Distance teaching universities and regional strategic development (Roger Mills, The Open University in East Anglia, Reino Unido). Public-private partnerships in the massification of open and distance tertiary study (Douglas G. Shepherd, Central Queensland University, Australia). Contribution of distance education to democratizing learning: a model to be evaluated (Fenando Bolaños, UNED, Costa Rica). Distance education and the reform of university education in Germany (Hannes K. Lehmann, Technische Universität Dresden, Alemania). Competition and partnership elements in a strategy for a modern distance learning establishment (Michel Moreau, Centre National d'Enseignement á Distance, Francia). Bridging the economic divide: the role of international alliances and educational technology in mediating soft-skills training for Latin America. A case study (Jean F. Pritchard y otros, Penn State University/CREAD, Estados Unidos).

Las enormes potencialidades de la Educación a Distancia para impulsar el Desarrollo Regional, Nacional o Internacional, que ya habían sido esbozadas en el Tema anterior (c) sobre Globalización, encuentran en el conjunto de trabajos presentados en este Tema (d), una expresión de su gran amplitud y diversidad. Desde el empleo de la Educación a Distancia para la Reestructuración de las Universidades Latinoamericanas, hasta la creación de Redes de Aprendizaje para Europa, ó en las campañas para la democratización de las sociedades latinoamericanas, o la Reforma de la Universidad Alemana, estos casos representan sólo algunos de los ejemplos del extraordinario alcance de esta moderna Modalidad. Sin embargo, y para el logro de estos ambiciosos propósitos, la Educación a Distancia requiere combinarse con las Nuevas Tecnologías y con avanzados procesos e instrumentos de Planificación Estratégica.

En relación al Tema (e) "Demoliendo las barreras mediante educación y entrenamiento", mencionaremos los trabajos siguientes: Higher education as a means of breaking down social barriers. The Open University of Israel and its Arab students (Yael Enoch y otros, The Open University of Israel, Israel). Breaking down social and cultural barriers which impair the progress of students in higher and distance education (Margaret Lourens, Technikon Southern Africa, Suráfrica). Empowerment of the disadvantaged through open learning and training: the IGNOU experiment (Kulandaiswamy Soundravalli, Indira Gandhi National Open University, India). Organizational culture studies: an urgent need to understand distance universities management in Latin America (Aleska Cordero, Universidad Nacional Abierta, Venezuela). Enhancing women's access to higher education in Nigeria through distance education (Emma Obasi, Imo State University, Nigeria). Breaking down barriers through education and training - Gender Issues (Ajaib Singh y otros, Panjab University, India). Genre, woman in distance education (Martha Inés Solano, Pontificia Universidad Javeriana, Colombia). Distance learning as a tool for rural development (Kerstin Fagerstrom y otros, Abo Akadami University, Finlandia). Caught between social pressure and the final barrier (Sakom Boondao, Sukhothai Thammathirat Open University, Tailandia). Impact of crosscultural differences in distance teaching overseas (Victoria Libin y otros, The Open University of Israel, Israel). Recruitment and study barriers in the electronic college - revisited (Torstein Rekkedal, NKI Distance Education, Noruega). Distance education: a paradigm for the development of intercultural contexts (Cristina Simmonds, Universidad del Cauca, Colombia).

Se percibe al examinar varios de los trabajos presentados para este Tema (e), que sus autores consideran que esta Modalidad puede proporcionar ayudas significativas también en la difícil tarea de romper barreras sociales, culturales, y raciales, que generalmente impiden la integración democrática de sociedades y grupos. En el caso de países de poco desarrollo, el problema se refiere principalmente a los sectores rurales y al desigual status de la mujer.

Finalmente, el Tema (f) "Mercados y Mercadeo", por ser un tema relativamente nuevo para muchas universidades, se compone de un pequeño numero de trabajos: Using new technologies to extend the boundaries of distance taught management development (C.W.J.A. Gray, The Open University Business School, Open University, Reino Unido). Marketing the virtual university: from local to global (Jannette Kirkwood y otros, The University of Southern Queensland, Australia). Change in cost-effectiveness of the University of the Air of Japan with its nationwide expansion (Hiromitsu Muta y otros, Tokyo Institute of Technology, Japón). An academic exchange market for multimedia components (Uwe Steinmann, FernUniversität Hagen, Alemania). An international virtual seminar for university faculty and administrators. Professional development in distance education - A successful experiment and future directions (Ulrich Bernath y otros, Carl von Ossietzky University of Oldenburg, Alemania). Distance education and job market: a case study of IGNOU graduates 
(Ashok Kumar Gaba, India). Developing the work-force of the petroleum industry through virtual learning (Fabio J. Chacón, International Center for Education and Development, Venezuela).

En un mundo globalizado, con importantes y continuos cambios, cualquier Universidad del futuro, encontrará cada vez más, un amplio y cambiante radio de acción, que muchas veces supera la Región y el País, que supuestamente debían ser sus Zonas de Influencia; en esta extensa zona operan también otras universidades e instituciones no universitarias, que ofrecen igualmente, enseñanza, investigación y servicios a la comunidad, ocasionándose muchas veces solapamiento de iguales ofertas. Por ello resulta necesario que la universidad esté preparada para comprender claramente los elementos que constituyen sus "ventajas competitivas".Es allí donde los conceptos de "mercado y mercadeo"resultan cada vez más importantes a pesar de que algunos académicos tradicionales suelen ver como de mal gusto, introducir en la universidad tales conceptos, que ellos consideran sólo apropiados para actividades industriales y mercantiles. Todo esto requiere una concepción moderna de la Gerencia Universitaria, que tiene una especial utilidad para universidades y mega-universidades a distancia. Los trabajos señalados en el párrafo anterior, proporcionan alguna indicación sobre los diferentes enfoques que pueden tomar el estudio de este tema.

\section{ICDE: VANCOUVER (1982) VERSUS VIENA (1999)}

En los diecisiete años transcurridos desde la Conferencia ICDE Vancouver (1982) hasta la actualidad ICDE Viena (1999) se han producido incesantemente, numerosos e importantes cambios, tanto en el amplio contexto socio-cultural-económico, donde se inserta y desarrolla la educación a distancia, como en el interior de la estructura y procesos de esta nueva modalidad. El ritmo de estos cambios ha sido generalmente tan acelerado que muchos directivos y académicos de las universidades a distancia existentes, enfrentan serias limitaciones para visualizarlos objetivamente, pero más importante aún, encuentran dificultades para lograr entender y aceptar, en que forma tales cambios deberían generar respuestas innovadoras y eficientes en sus respectivas instituciones.

Para examinar con mayor objetividad la naturaleza de tales cambios, los dividiremos en tres categorías: los provenientes del contexto Nacional y Mundial; los relativos a la estructuración de las nuevas universidades; y, los relativos a los procesos institucionales internos de aprendizaje, investigación y extensión.

\subsection{LOS CONTEXTOS NACIONAL Y MUNDIAL}

En la época en que se realizó la Conferencia Vancouver (1982) los límites del campo de acción de cada universidad se encontraban bastante definidos. Para las Universidades Presenciales, era principalmente su "campus" y a lo sumo, unas pocas extensiones en alguna localidad no muy lejana. En cambio, para las Universidades a Distancia de aquel tiempo, ese campo de acción se limitaba generalmente a un Sector, Región ó a su País. Por otra parte, las influencias externas que recibía la universidad, provenían también de la Región o del País respectivo. En contraste, hoy día, tanto las fuerzas que provienen del exterior, como la dimensión del territorio que ella puede abarcar con sus servicios y ofertas académicas, tiende a ampliarse mucho más allá de cualquier frontera nacional. Examinemos por consiguiente, cuales son algunas de estas fuerzas que están moldeando a las futuras universidades a distancia:

\subsubsection{Globalización}

Este es un fenómeno mundial moderno que tiende hacia la interconexión a escala mundial, de aspectos económicos, culturales, socio-políticos, informativos, tecnológicos, educacionales, etc. Resulta hoy día más evidente esta condición en lo económico, donde una expresión muy concreta son las compañías multinacionales. Sin embargo, nuestro interés se centra aquí en la "globalización educativa", porque ella influye poderosamente en la organización, operación y funciones académicas de las universidades a distancia. Las ventajas de poder ofrecer cursos y programas transnacionales de alta calidad, flexible acceso y participación para estudiantes de diversos países, y una óptima utilización de los mejores recursos intelectuales y académicos mundiales, constituyen sólo algunos de los elementos a considerar en los nuevos diseños curriculares e institucionales. Por otra parte, debemos alertar aquí sobre el peligro de que la globalización conduzca hacia una exagerada homogenización de la cultura, en detrimento de culturas regionales y nacionales autóctonas. Sin duda, el fenómeno de la Globalización ha adquirido ahora una destacada importancia que no se visualizó en Vancouver (1982).

\subsubsection{Sociedades cada vez más informatizadas}

Todas las sociedades son ahora o tenderán a serlo en un futuro muy cercano, "sociedades basadas en el conocimiento y en el aprendizaje". Una sociedad informatizada exige que sus universidades investiguen las implicaciones de ese cambio tan 
radical y modifiquen sustancialmente sus estructuras y los enfoques curriculares para formar a los intelectuales, profesionales y líderes que deberán dirigir y actuar en esta sociedad tan diferente. Aunque para la fecha en que se celebró Vancouver (1982) el proceso de informatización ya se percibía inicialmente en varios países representados en la Conferencia, ha sido en el período siguiente hasta hoy, cuando el desarrollo de la informatización mundial ha experimentado extraordinarios avances, en casi todos los sectores de la mayoría de las sociedades contemporáneas.

\subsubsection{Educación Permanente}

Desde hace mucho tiempo los ciclos de vida de un ciudadano normal se diferenciaban mediante tres etapas: "escuelatrabajo-retiro". Sin embargo, hoy día, en un mundo sujeto a constantes cambios en lo social, lo político, lo económico, y lo tecnológico, ya no resulta suficiente estudiar solamente en la primera etapa, pues la renovación del conocimiento y la adquisición de diferentes destrezas, requieren de un aprendizaje de por vida. Esta nueva condición y tendencia, que todavía no resultaba tan evidente en el año en que se celebró Vancouver (1982), obliga ahora a reconsiderar la extensión y la diversidad de las ofertas académicas de las futuras Universidades a Distancia.

\subsubsection{Formación y Entrenamiento para el Trabajo y la Producción}

La capacitación y actualización, directamente relacionadas con el Trabajo y la Producción, (Industrial, Comercial y de Servicios) está adquiriendo cada vez más importancia para las universidades a distancia. En el caso de la Open University y otras instituciones del Reino Unido, esta nueva función prácticamente ignorada en Vancouver (1982), se denomina ahora generalmente como "Open Learning para Sectores Productivos". En la Conferencia de Viena (1999) y respondiendo al vigoroso desarrollo mundial de esta especial oferta académica, se le dedicó uno de los tres ejes principales: "(T): Desarrollando las Compañías y Entrenando su Fuerza de Trabajo".

\subsubsection{Mercadeo y Mercados}

Este es uno de los nuevos temas que no fue considerado anteriormente en la reunión de Vancouver (1982). Aunque ahora en Viena (1999), no recibió una atención muy destacada, sin embargo es evidente que la universidad frente a sus posibles mercados cambiantes, el tema está adquiriendo cada vez más importancia, tal como puede observarse en la referencia específica hecha en este informe.

\subsubsection{Nuevas Tecnologías}

El vertiginoso desarrollo de la Computación, de la Telemática y de la Informática en general, han significado para la Modalidad a Distancia cambios trascendentales, extensamente documentados en todas las actividades y sesiones de la Conferencia Viena (1999). La enorme importancia de tales cambios obligan a repensar el Paradigma inicial que fue el que sirvió de base para las discusiones de Vancouver (1982). En tal sentido, el presente informe permite visualizar las posibles orientaciones y alcances de un nuevo Paradigma "Informático-Telemático", cuya implantación no debe posponerse.

\subsection{NUEVOS MODELOS Y ESTRUCTURAS}

La debida consideración de las nuevas fuerzas externas que gravitan sobre las universidades a distancia, existentes o en proyecto, obligan a considerar cuales deberán ser los Modelos y Estructuras más apropiados para contener las funciones básicas y las nuevas que requerirán estas universidades. Resulta evidente que se trata de un proceso complejo y trascendental, quizás más difícil en muchas de las universidades a distancia existentes, las cuales en muchos casos, iniciaron sus actividades enmarcándolas en estructuras universitarias tradicionales, y sin tomar muy en cuenta que la operación de una universidad a distancia diferiría, cada vez más, de la de una universidad presencial tradicional. Para estudiar este delicado problema, que tiene que ver con todos los seis Temas Principales del "Eje (U): Construyendo la Universidad del Futuro", examinaremos los aspectos siguientes:

\subsubsection{Modelos y Estructuras}

En general, la idea generatriz de los nuevos modelos, gira alrededor de dos conceptos interconectados: (a) que el aprendizaje es más importante que la enseñanza y que por consiguiente, el proceso instruccional debe estar cada vez más centrado en el estudiante; y (b) que el uso funcional de las nuevas tecnologías, posibilita distintos tipos de interactividad, que enriquecen el 
aprendizaje individualizado y sus costos de operación. Sólo como dos ejemplos de muchos modelos posibles, presentaremos el de Taylor (1999) y un Modelo de Universidad Virtual, ejemplificado por la "Universitat Oberta de Catalunya".

Las cuatro primeras Generaciones descritas en el Cuadro que sigue de Taylor (1999), se refieren a una clasificación usada generalmente, en la Educación a Distancia. Puede considerarse que hoy día, muchas universidades a distancia (especialmente de países de poco desarrollo), están ubicadas principalmente en el Modelo titulado de Segunda Generación, pero iniciándose modestamente, en el uso de algunos elementos del Modelo de Tercera Generación. Lo que caracteriza aquí a la Quinta Generación, y su diferencia con la Cuarta, según Taylor, es que la Quinta introduce el "Sistema de Respuestas Automatizadas", lo cual presenta un gran potencial para el logro de importantes economías de escala, especialmente para el manejo moderno de los apoyos académicos y de enseñanza.

En lo relativo al ejemplo de un modelo existente de Universidad Virtual, se presentó en Viena (1999) a través de diversos trabajos, la "Universitat Oberta de Catalunya". Esta es una universidad de reciente fundación, con un radio de acción circunscrito a la Región Catalana y promovida por el gobierno de esa región. Al examinarse los documentos pertinentes, se observa que la "virtualización " de esa universidad responde a una concepción integral, centrada en el aprendizaje del estudiante y en el uso cuidadosamente coordinado de las tecnologías más avanzadas, aplicadas a esta Modalidad educativa. Lo virtual se apoya en un "campus virtual", las bibliotecas virtuales, los sistemas de comunicaciones mediante Intranet, investigaciones sobre lo "virtual", formación y orientación especial de profesores, plataformas para usuarios, plataforma de servidores, etc. El ejemplo de esta institución totalmente virtual, que a pesar de sus pocos años fue galardonada recientemente con el "Bangemann Challenge Prize" de la Comisión Europea,( como la mejor iniciativa telemática en Educación a Distancia), contrasta con la tendencia actual de algunas universidades, especialmente de países de bajo desarrollo, de autoproclamarse como Universidades Virtuales, por el mero hecho de adicionar a su enseñanza esencialmente tradicional, alguna tecnología audiovisual.

Los Modelos para la Educación a Distancia constituyen un punto de partida importante para el diseño posterior de innovadoras "Estructuras Organizativas" que permitan llevar a cabo con eficiencia y eficacia las funciones, programas y tareas correspondientes. En este sentido están en marcha diversos ensayos de "Reestructuración Institucional" congruentes con distintas estructuras provenientes a veces, del área de negocios pero representando esquemas muy diferentes a los que conocemos como estructuras universitarias convencionales (véase Moore, 1999). Los aspectos organizacionales, incluyendo la Evaluación Institucional, curiosamente recibieron muy poca atención, tanto en Vancouver (1982) como en Viena (1999).

Tabla 1: Modelos de Educación a Distancia. Una Estructura Conceptual. Taylor (1999) (pp. 3-4)

\section{MODELOS DE EDUCACIÓN A DISTANCIA}

\begin{tabular}{|c|c|c|}
\hline $1^{\text {a }}$ Generación & El Modelo de Correspondencia & Ue Textos Impresos \\
\hline $2^{\mathrm{a}}$ Generacion & El Modelo Multi-media & 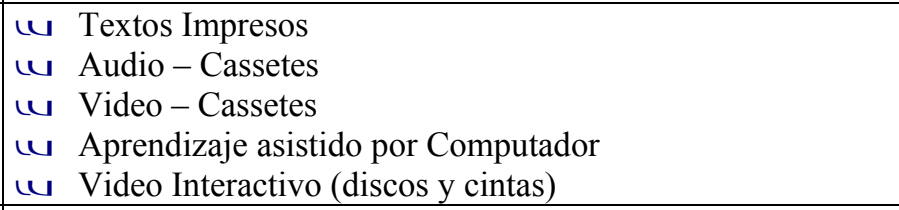 \\
\hline $3^{\text {a }}$ Generación & El Modelo de Teleaprendizaje & $\begin{array}{ll}\text { ur } & \text { Audioteleconferencia } \\
\text { ur } & \text { Videoconferencia } \\
\text { ur } & \text { Comunicación Audiográfica } \\
\varkappa & \text { Transmisiones TV/Radio y Audio Teleconferencia } \\
\end{array}$ \\
\hline $4^{\text {a }}$ Generación & El Modelo Flexible de Aprendizaje & \begin{tabular}{|ll} 
Uw & Multimedia Interactivo (IMM) \\
w & Acceso a Recursos de WWW basados en Internet \\
we & Comunicaciones mediadas por Computador
\end{tabular} \\
\hline $5^{\text {a }}$ Generación & $\begin{array}{lrl}\text { El Modelo } & \text { Flexible-Inteligente de } \\
\text { Aprendizaje } & \end{array}$ & $\begin{array}{l}\text { Uu } \text { Multimedia Interactivo (IMM) } \\
\text { ur Acceso a Recursos de WWW basados en Internet } \\
\text { ur Comunicaciones mediadas por Computador, usando } \\
\text { sistema de respuestas automatizadas }\end{array}$ \\
\hline
\end{tabular}




\subsection{APRENDIZAJE, INVESTIGACIÓN Y EXTENSIÓN}

Estas tres funciones, y sus componentes que resultan esenciales para cualquier universidad a distancia, son las que han acusado mayores cambios, en la modalidad a distancia, si comparamos su situación actual, reflejada en la Conferencia de Viena (1999), con el panorama que presentaba la Conferencia de Vancouver (1982). A continuación, destacaremos, solamente, algunos de los aspectos que requieren mayor atención:

\subsubsection{Bases Teóricas}

De Vancouver (1982) a esta fecha se han producido importantes progresos en lo relativo a precisar los fundamentos teóricos que ayudan a explicar y a desarrollar los aspectos prácticos de la Educación a Distancia. Especialmente importantes han sido los aportes de Holmberg (1989), Keegan (1993), Moore (1996) y más recientemente, Simonson y otros (1999). Sin embargo, en la Conferencia de Viena (1999), concurrieron muy pocos trabajos relativos a este importante tema, posiblemente, porque muchos educadores consideraron que ya existía suficiente claridad al respecto y que por otra parte, un buen número de los trabajos presentados se fundamentaron explícita ó implícitamente, en las teorías investigadas y aceptadas.

\subsubsection{Medios}

La extraordinaria cantidad y diversidad de nuevos "Medios Instruccionales" y la necesidad de combinarlos funcionalmente para obtener los objetivos del aprendizaje, han hecho surgir especialidades que faciliten su harmonización e inserción. Este es el caso de la "Mediática" y la "Telemediática", surgidas especialmente en Francia y Canadá, y en Inglaterra, con el título de "Medios para el Conocimiento" ("Knowledge Media"). Los numerosos trabajos presentados en Viena (1999) utilizando una gran variedad de multimedios, demuestran la importancia de estas nuevas especialidades.

\subsubsection{Educación Abierta y Educación a Distancia}

Aunque estos dos conceptos no son similares, su interconexión está resultando cada vez más útil. En efecto, la Educación Abierta facilita que la Modalidad a Distancia, se centre más en el alumno y su aprendizaje. En este sentido, los enormes avances de las Tecnologías Informática-Telemática posibilitan cada vez más, la "individualización del aprendizaje", cuestión esta que siempre ha figurado entre los objetivos de la Educación a Distancia, pero que ha tropezado con la rigidez tradicional de muchos materiales de instrucción, que por razones de costo han tendido hacia productos standard e iguales para todos los alumnos.

\subsubsection{Investigación}

Como se ha expresado en Secciones anteriores de este Documento, las numerosas investigaciones, estudios y publicaciones producidas desde Vancouver (1982) hasta nuestros días han ayudado a consolidar y legitimar la Modalidad a Distancia, aunque todavía existen en todo el mundo, áreas de esta especialidad que requieren mayor estudio e indagación. El conjunto de trabajos de Viena (1999) reflejan que los mayores aportes para la investigación provienen generalmente de Alemania, Australia, Canadá, España, y Estados Unidos, Dentro de este Sector de Investigaciones, todo parece indicar la necesidad de extender rápida y masivamente, los procesos de formación y actualización de Investigadores, Docentes, Tutores y Técnicos, porque en las universidades a distancia, todavía existen grupos muy importantes de sus académicos provenientes de la Educación Presencial, enfrentando dificultades para su efectiva transición y adaptación.

\subsubsection{Estudiantes}

Las consideraciones hechas en apartes anteriores de este trabajo, demuestran las tendencias actuales hacia la individualización del aprendizaje y hacia el empleo e integración cada vez más eficiente de las nuevas tecnologías. En este sentido, la irrupción reciente de los novedosos conceptos de "Aprendizaje Virtual" y "de "Virtualización " de las Universidades, despertaron extraordinaria atención en las deliberaciones de la Conferencia Viena (1999).

\subsubsection{Diseño y Producción de Materiales Instruccionales}

Muchos de los enormes cambios de la Educación a Distancia, producidos durante el lapso comprendido entre Vancouver (1982) y Viena (1999), repercuten especialmente en el Diseño y Producción de Materiales, así como en los Apoyos Académicos requeridos durante el Proceso de Aprendizaje. En relación con los primeros, resulta evidente que hoy día no es 
necesario para cada institución, diseñar y producir desde" cero" todos y cada uno de los cursos e instrumentos que requiere un Programa determinado, porque aunque ello pueda ser teóricamente deseable, pocas universidades ( y especialmente las de países de bajo desarrollo), pueden incurrir en los astronómicos costos en términos de: recursos profesionales, tiempos y financiación, que exige la producción total. Por otra parte, la situación de hoy no es igual a la de la Década de los años 80 , signada por una limitadísima oferta de Cursos disponibles de calidad, lo cual obligaba a las instituciones a enfrentar toda su producción, a pesar de las limitaciones ya mencionados. Así mismo, la utilización en gran escala de Nuevas Tecnologías y las condiciones de Globalización e Informatización ya señaladas, permiten concebir la solución de este problema mediante formas y con instrumentos, que permitan calidad, pertinencia y bajos costos. Finalmente, en relación con los Apoyos Académicos, aquí también, mediante las Tecnologías actuales de Informática y especialmente la Telemática, pueden cambiarse radicalmente, los procesos tradicionalmente ineficientes y costosos de Tutoría y Asesoría.

\section{SÍNTESIS DE CONCLUSIONES}

El examen y comparación de las características y diferencias de la Educación a Distancia, observadas entre las Conferencias del ICDE, de Vancouver (1982) y Viena (1999), revela que en los diecisiete años transcurridos, esta Modalidad a Distancia ha experimentado trascendentales y continuos progresos relativos: a sus Modelos y Estructuras Organizacionales; a sus Fundamentos Teóricos e Investigaciones; a sus alcances territoriales, que se han extendido a otras esferas diferentes a la inicial, limitada a Universidades a Distancia; a nuevos enfoques de Diseño y Formas de Producción de Materiales; a Medios más efectivos de Comunicación, Tutorías, y Evaluación; y en general, a una amplia y efectiva utilización de nuevas Tecnologías Informativas-Telemáticas. Por otra parte, algunas Temáticas que no se vislumbraron en Vancouver (1982) aparecieron ahora en Temas como: Globalización; Políticas y Estrategias para el Desarrollo; Aprendizaje Virtual y Universidades Virtuales; Educación y Entrenamiento para el Trabajo y la Producción; y, Mercados y Mercadeo.

Dentro del conjunto de antiguos y nuevos temas, los dos que aparecieron como los más dominantes en Viena (1999) fueron el de las Nuevas Tecnologías y el relativo a la Virtualización de las Universidades. Por otra parte, se apreció el enorme impacto que está produciendo la Educación a Distancia como instrumento vital para impulsar: Procesos de Desarrollo de Regiones y Países; la transformación de Universidades Presenciales; campañas de Democratización Ciudadana; Escuelas y Liceos, donde la Masificación requiere respuestas masivas de alta calidad educativa y bajos costos. Así mismo, pudo constatarse, el decisivo papel instrumental de los conceptos de: "Aprendizaje", "Diseño", "Investigación", e "Interactividad", en todos los procesos de aplicación y modernización de la Modalidad a Distancia.

El vigoroso crecimiento de la Educación a Distancia, observado tanto en países de gran desarrollo como en países de bajo desarrollo, no está generado por "Modas" o "Imitaciones" momentáneas, sino por la necesidad de responder ventajosamente, a nuevas necesidades de las sociedades contemporáneas. En efecto, la Educación a Distancia permite: llevar el conocimiento a donde están los que lo desean y necesitan; a dar oportunidades educativas a personas que no las encontraban en acreditadas instituciones tradicionales; a poder armonizar "estudios-trabajos-familia"; reconocer la necesidad actual de continuar aprendiendo durante toda la vida; de tener acceso a estudios de alta calidad y de bajo costo; y, finalmente, de lograr un aprendizaje cada vez más individualizado, y relevante, para las condiciones y aspiraciones de cada estudiante. Sin embargo, para que la Educación a Distancia alcance tan importantes objetivos, no se producirá por arte de magia y requerirá un exigente y continuo proceso de planificación, estructuración, organización, operación y evaluación, que aseguren Calidad, Innovación y Pertinencia. De no cumplirse tales rigurosas condiciones, la Educación a Distancia podría convertirse en un gigantesco obstáculo para cualquier forma de desarrollo, pues la concesión masiva de títulos y certificados que no impliquen efectiva adquisición de conocimientos, y de una verdadera educación, no representarían contribución alguna, ni al progreso de la sociedad, ni al de cada uno de los ciudadanos.

El extraordinario potencial que se observó en la Conferencia Viena (1999) para la Educación a Distancia y las Nuevas Tecnologías, plantea un reto exigente e inaplazable para muchas universidades a distancia, existentes o en proyecto. En efecto y como hemos podido observar en el presente documento, los cambios y progresos para la Modalidad y sus Tecnologías de apoyo, tienen un enorme dinamismo, que seguramente continuará y aumentará en el próximo milenio. Sin embargo, las universidades que carezcan de la capacidad y disposición para renovarse con la velocidad necesaria para estos grandes cambios, no podrán refugiarse en la suposición de que el sólo hecho de denominarse universidades a distancia resultará suficiente para producir y obtener los enormes beneficios y ventajas de esta nueva Modalidad a Distancia. En el convulsionado mundo actual, las alternativas para estas universidades y programas son muy pocas: renovarse a fondo y a tiempo o fracasar como instituciones capaces de influir en los avances sociales y científicos.

\section{REFERENCIAS BIBLIOGRÁFICAS}


SECCION: ICDE / VANCOUVER 82,

Hito significativo en la evolución de la Modalidad a Distancia

NOTA:

Todas las citas mencionadas en esta Sección, con excepción de la de Wedemeyer (1981), están contenidas en la Publicación de los Proceedings de esta Conferencia, con el titulo: Learning at a Distance: A World Perspective. (1982) Eds. John S. Daniel, Martha A. Stroud, y John R. Thompson. Athabasca University. Edmonton.

WEDEMEYER, C. A. (1981). Learning at the back door: reflections on non-traditional learning in the lifespan. The University of Wisconsin Press.

SECCION: ICDE /VIENA 99

La Nueva Frontera Educativa. Enseñando y Aprendiendo en un Mundo en Red

NOTA:

Todas las citas mencionadas en esta Sección, con excepción de la de Naughton (1994), están contenidas en los Proceedings de esta Conferencia, publicados mediante un CD-ROOM del ICDE Viena, Junio 20-24, 1999. Oslo.

NAughton, J. (1994). What is Technology, en Teaching Technology. Ed. Frank Banks. London: The Open University/Routledge.

SECCIONES: ICDE: VANCOUVER (1982) versus VIENA (1999) / SINTESIS DE CONCLUSIONES.

HOLMBERG, B. (1989). Theory and practice of distance education. London: Routledge.

KEEGAN, D. (ed.) (1993). Theoretical principles of distance education. London: Routledge.

MoORE, M. G. \& KinSLEY G. (1996). Distance education: a systems view. New York: Wadsworth Pub.

Moore, M. G. (1999). Institutional restructuring: is distance education like retailing? en The American Journal of distance education, 13 (1). University

Park: The Pennsylvania State University.

Simonson, M., Schlosser, C., And Hanson, D. (1999). Theory and distance education: a new discussion, en The American Journal of Distance Education, 13 (1). University Park: The Pennsylvania State University.

\section{PERFIL ACADEMICO DE LOS AUTORES}

Miguel Casas Armengol es Arquitecto: Universidad Nacional Colombia; Universidad Central Venezuela. Ph.D. en Educación: Stanford University. California.US. Prof. Titular: Universidad del Zulia. Maracaibo / Universidad Nacional Abierta. Caracas. Decano-Fundador Fac.Arquitectura. Universidad del Zulia. Rector-Fundador Universidad Nacional Abierta. Investigador Visitante: del Instituto de Planificación Educativa. UNESCO.Paris; y de la Stanford University.

Autor de "Universidad sin Clases" y Coautor (capítulos) en siete libros de Pergamon Press y UNESCO.

Líneas de Investigación: Universidad y Desarrollo Nacional/Regional. Organización y Modernización de Universidades a Distancia. Nuevas Tecnologías en Educación Superior a Distancia.

Dirección electrónica: masas@eldish.net.

Fax: (582) 793.68.02

Lily Stojanovic es Educadora, egresada de la Universidad Central de Venezuela (UCV). Tiene un M.A. en Curriculum de la Stanford University, California. U.S. Prof. Titular UCV y UNA (Universidad Nacional Abierta). En Venezuela ha sido Pionera de los Estudios a Distancia: desde 1972 en los Estudios Universitarios Supervisados en la UCV; y posteriormente, desde 1978, en la UNA. Ha publicado un libro sobre Evaluación Estudiantil en Sistemas a Distancia y otro sobre Evaluación del Material Instruccional, también para programas a Distancia.

Dirección electrónica: 1ilystojanovic@yahoo.com 